\title{
Aumento de las resistencias del cemento mediante la formación adicional de ettringita
}

La ettringita puede formarse a partir de diferentes materias primas, pero sus tres componentes: $\mathrm{CaO}, \mathrm{SO}_{3}$ y $\mathrm{Al}_{2} \mathrm{O}_{3}$ deben encontrarse con la suficiente concentración en fase líquida para permitir su formación.

El método de preparación de ettringita es conocido desde W. Michaelis (1); posteriormente fue utilizado por varios investigadores. En este método se parte de una disolución de sulfato de aluminio y cal, con o sin adición de yeso. La ettringita así obtenida es idéntica a la formada mediante la hidratación de los minerales del clínker en presencia del yeso (2).

Es necesario procurar que se produzcan paulatinamente los componentes de cal para que se efectúe de manera predominante la formación de ettringita, después de haberse amasado y enmoldado el mortero de cemento. Sólo así, en régimen de reposo de la masa, se puede constituir un esqueleto útil para la estructura y no cristales aislados sin enlace. Para este objeto es muy adecuado el hidróxido cálcico formado durante la hidratación del cemento. Su acción mutua con el sulfato de aluminio, que es preciso añadir en forma de disolución, se puede representar en la siguiente ecuación:

$$
\mathrm{Ca}(\mathrm{OH})_{2}+\mathrm{Al}_{2}\left(\mathrm{SO}_{4}\right)_{3}+25 \mathrm{H}_{2} \mathrm{O}=3 \mathrm{CaO} \cdot \mathrm{Al}_{2} \mathrm{O}_{3} \cdot 3 \mathrm{CaSO}_{4} \cdot 31 \mathrm{H}_{2} \mathrm{O} \text {. }
$$

Con esta reacción se comprobó la influencia favorable del cloruro de calcio para el aumento de resistencia del cemento. Para impedir una acción mutua entre dichos aditivos en mayor medida, se disuelven ambos por separado, en pequeñas cantidades, en el agua de amasado.

En opinión de algunos autores (3) el cloruro cálcico destruye la ettringita, pero la mayor parte de ellos opinan que no actúa en forma destructiva, si no que hasta contribuye a obtener el hidrato del sulfoaluminato cálcico (4-7).

Bajo la influencia del aditivo a base de sulfato de aluminio y de cloruro cálcico se produce, durante el primer período de fraguado, un grado más avanzado de hidratación.

Con objeto de impedir que no se origine un fraguado demasiado rápido, una fisuración del cemento y un aumento de corrosión de las armaduras de acero, se usó el aditivo compuesto en pequeñas cantidades.

Hay que citar también otras investigaciones, en las que se utilizó una adición mayor de sulfato de aluminio solo o junto con polvo de aluminio, lejía residual de sulfito, cloruro cálcico (8) o con polvo de aluminio, lejía residual de sulfito, nitrato cálcico, nitrito de calcio (8) o con alumbre, sulfato cálcico, hidróxido de aluminio (10), para preparar cementos expansivos. 


\section{Materias primas}

La composición mineralógica de los cementos aparece en la tabla 1 . La cantidad de yeso alcanzó aproximadamente el $2 \%$, calculado como $\mathrm{SO}_{3}$. El cemento III se almacenó durante unos tres meses.

La arena de cuarzo estándar tenía un contenido de $\mathrm{SiO}_{2}$ de más de $99 \%$ y una granulación de 0,74 a $1,39 \mathrm{~mm}$.

El sulfato de aluminio y el cloruro cálcico eran productos técnicos.

TAB L A 1

Composición mineralógica de los cementos

\begin{tabular}{|c|c|c|c|c|}
\hline & \multicolumn{4}{|c|}{ Composición en $\%$ ponderal } \\
\cline { 2 - 5 } & $\mathbf{C}_{3} \mathbf{S}$ & $\mathbf{C}_{2} \mathbf{S}$ & $\mathbf{C}_{3} \mathbf{A}$ & $\mathbf{C}_{4} \mathbf{A F}$ \\
\hline Designación & & & & \\
\hline Z I & 64,30 & 15,32 & 7,61 & 9,60 \\
Z II & 66,40 & 13,33 & 5,83 & 9,30 \\
Z III & 65,96 & 14,22 & 4,45 & 11,16 \\
\hline
\end{tabular}

\section{Composición del mortero de cemento y tipo de las probetas}

El tiempo de fraguado se determinó empleando pasta de cemento de consistencia normal y' se midió con el aparata Vicat. Las cantidades de agua para los cementos fueron de: $\mathrm{Z} \mathrm{I}=25 \%, \mathrm{Z}$ II $=28 \%, \mathrm{Z}$ III $=26 \%$.

La resistencia a compresión se comprobó preparando morteros de $1: 3$ con las cantidades de agua indicadas y se elaboraron cubos de $7 \mathrm{~cm}$ de acuerdo con las normas búlgaras para el cemento. Al cabo de 24 horas se extrajeron de los moldes y se conservaron al aire o bajo el agua.

La constancia de volumen se verificó sirviéndose igualmente de morteros $1: 3$, pero con $50 \%$ de agua, en moldes prismáticos de $4 \times 4 \times 16 \mathrm{~cm}$, cuyas variaciones longitudinales se midieron con el aparato Graf-Kaufmann. Los prismas se conservaron durante 48 horas al aire, hasta el séptimo día bajo agua y a continuación, en parte, al aire o bajo agua.

Para las investigaciones termográficas se molturaron parte de las probetas que se utilizaron para el fraguado. Después de conservadas, molturadas, al aire durante 10 a 360 días, se obtuvieron, con una velocidad de calentamiento de $10^{\circ} \mathrm{C} / \mathrm{min}$, las curvas del termoanálisis diferencial (DTA), de la termogravimetría derivada (DTG) y de la termogravimetría (TG).

La cantidad de los aditivos, calculados como $\mathrm{Al}_{2}\left(\mathrm{SO}_{4}\right)_{3}$ y $\mathrm{CaCl}_{2}$, alcanzó $0,7 \%$, aproximadamente, del peso de cemento de cada uno, o en total, $1,4 \%$.

Para averiguar las cantidades adicionales más adecuadas se exsayaron, también, los resultados con $1,2 \%$ de $\mathrm{Al}_{2}\left(\mathrm{SO}_{4}\right)_{3}$ y $1,2 \%$ de $\mathrm{CaCl}_{2}$, aproximadamente, o, en conjunto, $2,4 \%$.

Simplificando, las dos cantidades que están indicadas como aditivos son 1,4 y $2,4 \%$. 


\section{Tiempo de fraguado}

Los valores de los tiempos de fraguado figuran en la tabla 2 .

TAB L A 2

Tiempo de fraguado

\begin{tabular}{|c|c|c|c|c|}
\hline \multirow{2}{*}{ Composición } & \multicolumn{2}{|c|}{ Pricipio del fraguado } & \multicolumn{2}{|c|}{ Final del fraguado } \\
\hline & (Minutos) & (\%) & (Minutos) & (\%) \\
\hline 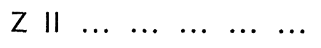 & 103 & 100,0 & 268 & 100,0 \\
\hline$Z$ II $+1,4 \%$ adición & 63 & 61,2 & 159 & 59,2 \\
\hline Z II $+2,4 \%$ adición & 58 & 56,3 & 96 & 35,8 \\
\hline
\end{tabular}

Al añadir el aditivo se observa aceleración del comienzo y principalmente del final del fraguado. Solamente la menor cantidad de aditivo cumple el requisito de la norma: "Comienzo del fraguado - no antes de una hora-.

\section{Resistencias iniciales}

Los valores medios de los seis cubos ensayados a la edad de un día se indican en la tabla 3 .

T A B L A 3

Resistencias a compresión de cubos a la edad de 1 día

\begin{tabular}{|c|c|c|}
\hline \multirow{2}{*}{ Cemento } & \multicolumn{2}{|c|}{ Resistencia media } \\
\hline & $\left(\mathbf{k p} / \mathbf{c m}^{2}\right)$ & $(\%)$ \\
\hline $\begin{array}{lllllll}z & \perp & \ldots & \ldots & \ldots & \ldots & \ldots\end{array}$ & 214 & 100 \\
\hline$Z$ I $+1,4 \%$ adición & 318 & 149 \\
\hline 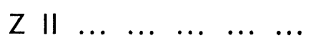 & 288 & 100 \\
\hline$Z \|+1,4 \%$ adición & 440 & 153 \\
\hline$Z \|+2,4 \%$ adición & 392 & 136 \\
\hline 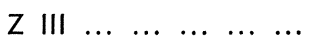 & 140 & 100 \\
\hline Z III $+1,4 \%$ adición & 288 & 206 \\
\hline Z III +2,4 \% adición & 259 & 185 \\
\hline
\end{tabular}

De la comparación de los resultados de los cementos I y II, que tienen diferentes contenidos de alita y aluminato, se puede deducir que la influencia del aditivo adicionado a un cemento con elevado contenido de alita es más favorable. Si se considera la reacción química [1] y se tiene en cuenta que el hidróxido de calcio procede principalmente de la alita, resulta comprensible el resultado.

La influencia del aditivo compuesto es más fuerte en el cemento a edades mayores. De los resultados relativos a los cementos II y III con contenido de alita bastante iguales, se deduce que el cemento III de más edad, con el aditivo compuesto, presenta una resis- 
tencia aproximadamente 1,35 veces superior. Esto muestra la influencia de la pequeña cantidad de aditivo para el aumento de la hidratación del cemento, especialmente en edades avanzadas.

\section{La importancia del factor agua/cemento (a/c)}

Los valores medios de resistencia de seis cubos con edad de 1 día y elaborados con el mismo cemento, pero con diferentes factores de agua/cemento, se muestran en la tabla 4.

\section{T A B L A 4}

Resistencias a compresión de cubos a la edad de 1 día

\begin{tabular}{|c|c|c|c|}
\hline \multirow{2}{*}{ Cemento } & \multirow{2}{*}{ Factor $a / c$} & \multicolumn{2}{|c|}{ Resistencia media } \\
\hline & & $\left(\mathbf{k p} / \mathbf{c m}^{2}\right)$ & $(\%)$ \\
\hline $\begin{array}{llllll}Z & \ldots & \ldots & \ldots & \ldots & \ldots\end{array}$ & 0,35 & 125 & 100 \\
\hline Z I $+1,4 \%$ adición & 0,35 & 281 & 225 \\
\hline $\begin{array}{lllllll}\mathrm{Z} \mid & \ldots & \ldots & \ldots & \ldots & \ldots\end{array}$ & 0,25 & 214 & 100 \\
\hline $\mathrm{ZI}+1,4 \%$ adición & 0,25 & 318 & 149 \\
\hline
\end{tabular}

Con un factor a/c superior, el aditivo compuesto produce un aumento mayor de resistencia. De aquí se puede deducir que las mezclas con más agua presentan mejores condiciones para el desarrollo de la estructura cristalina del hidrato de sulfoaluminato cálcico adicional formado con el aditivo y el aumento del grado de hidratación. Esto es muy importante en la práctica, porque las mezclas de hormigón y mortero contienen habitualmente mayores cantidades de agua de las que se requiere para la plasticidad normal.

\section{Aumento de resistencia y condiciones de almacenamiento}

Los aumentos de resistencia con el curado al aire y bajo el agua se presentan en las figuras 1 y 2 como valores medios de seis cubos.

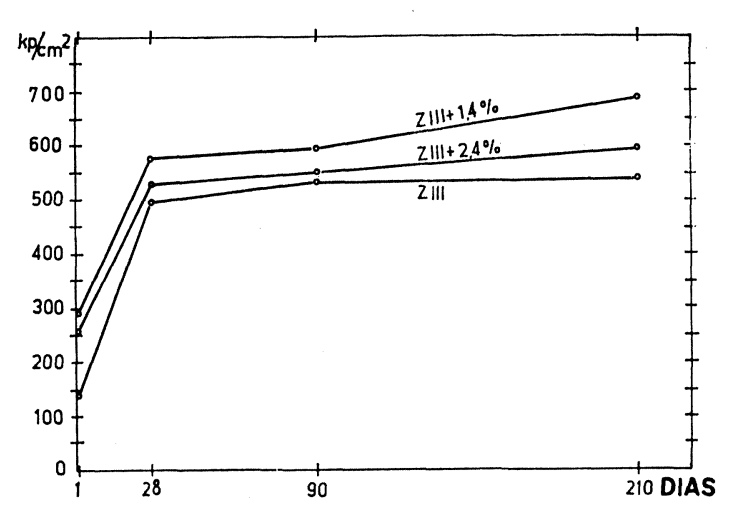

Fig. 1.- Resistencia a compresión al aumentar el tiempo del fraguado bajo el agua.

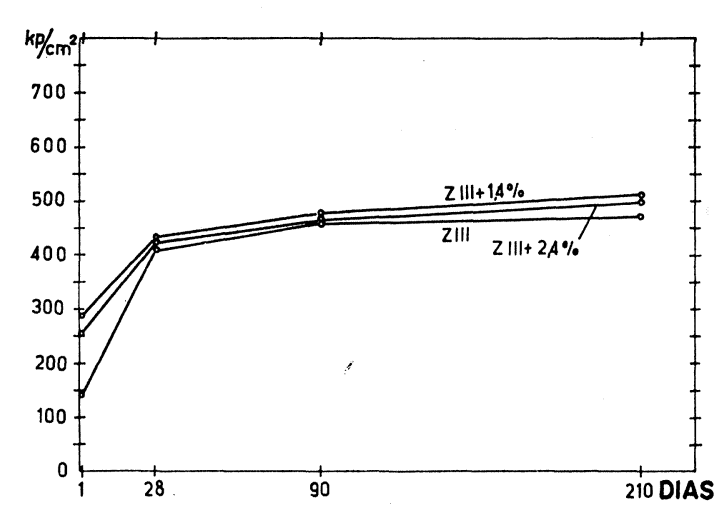

Fig. 2.-Resistencia a compresión al aumentar el tiempo de curado al aire. 
La adición formada por sulfato de aluminio y cloruro cálcico confiere al cemento portland normal propiedades de un cemento de endurecimiento rápido. Ya en el primer día, independientemente de las condiciones de fraguado, alcanza del 50 al $67 \%$ de la resistencia de 28 días y 58 al $70 \%$ de la resistencia a 28 días del cemento sin aditivo. Al incrementar el tiempo se reduce este aumento de resistencia, pero permanece, independientemente de las condiciones de curado, en el orden del 4 al $27 \%$.

Con la menor cantidad de aditivo se observan resistencias de 3 a $16 \%$ superiores.

\section{Constancia de volumen en función de la duración del fraguado}

Los valores medios de las variaciones de longitud de tres prismas, al aumentar el tiempo en función de las condiciones de curado, se pueden ver en las figuras 3 y 4.

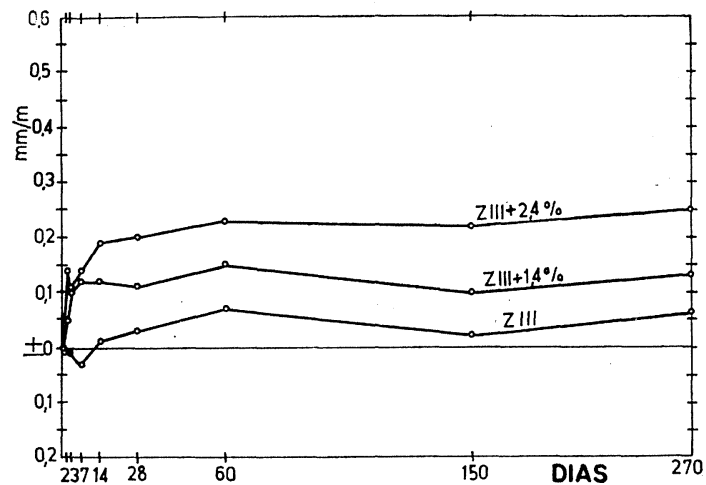

Fig. 3.-Aumento de volúmenes, en $\mathrm{mm} / \mathrm{m}$, al aumentar el tiempo de freguado bajo el agua.

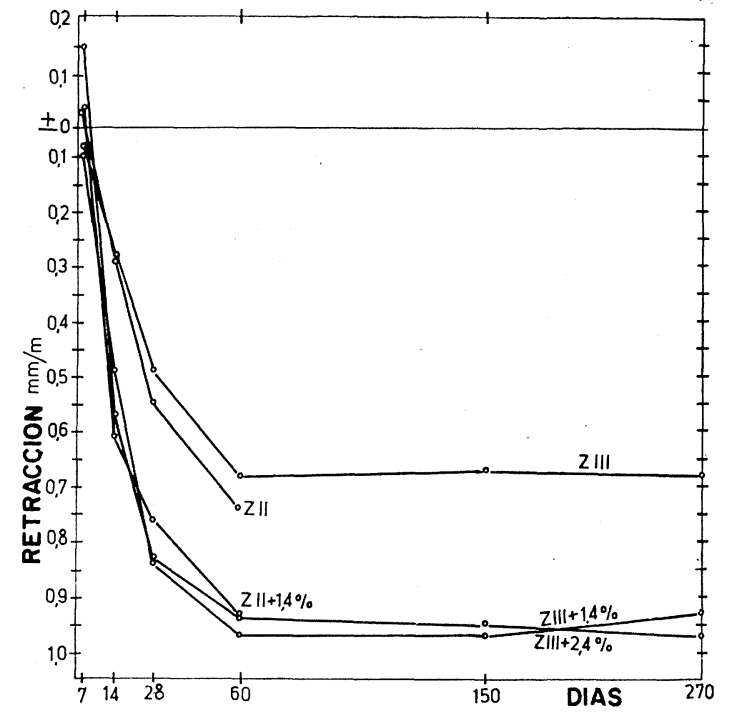

Fig. 4.-Retracción, en $\mathrm{mm} / \mathrm{m}$, al aumentar el tiempo de curado al aire.

Al curar bajo agua las probetas con el aditivo compuesto presentan un hinchamiento constante, al aumentar el tiempo, hasta llegar a los 60 días. Entonces disminuye el crecimiento y empieza a variar en el marco del valor alcanzado.

Si se supone el incremento de volumen del cemento sin aditivo igual a 100, las mezclas con aditivo compuesto, presentan, al cabo de 270 días, un crecimiento longitudinal de 217 con la menor cantidad de aditivo y 416 con la mayor. Evidentemente, ésta es una consecuencia de la cantidad de ettringita que se forma adicionalmente.

La ettringita se origina principalmente al empezar el fraguado; esto lo demuestran tanto las variaciones de volumen como los aumentos de resistencia. Las otras cantidades de ettringita, formadas al aumentar la edad del hormigón, son muy pequeñas y originan una compresión o retracción de la pasta de cemento. Esto confirma la lenta disminución y la supresión de la dilatación, así como también el continuo aumento de la resistencia con el tiempo.

Al fraguar al aire todas las probetas presentan una contracción que dura hasta unos 60 días; entonces las variaciones de longitud empiezan a ceder lentamente, y los prismas se estabilizan. 
Si se supone que la retracción de los cementos sin aditivo es igual a 100, la retracción aumenta al cabo de 60 días, en relación con $\mathrm{Z}$ II $+1,4 \%$ de aditivo, hasta 128 . En relación con $\mathrm{Z}$ III $+1,4 \%$ y Z III $+2,4 \%$ de aditivo, los valores de retracción alcanzan, al cabo de 270 días, 137 y 146, respectivamente.

Mediante el secado y la acción del ácido carbónico del aire en los cementos se producen variaciones de volumen. Los datos del ensayo muestran cierto aumento de la retracción debido a los aditivos. Este aumento es relativamente reducido, alcanzando del 28 al 37 $\%$ en relación con la pequeña cantidad de aditivo y no se manifiesta negativamente sobre la resistencia de la estructura. Cuando aumenta el tiempo. el cemento con aditivo conserva mayores resistencias que el cemento sin aditivo.

\section{Nuevas formaciones durante el fraguado}

Las curvas obtenidas por análisis termográfico, a una edad de 10 días, se muestran en las figuras 5,6 y 7 , y para la edad de 360 días, en las figuras 8 y 9.
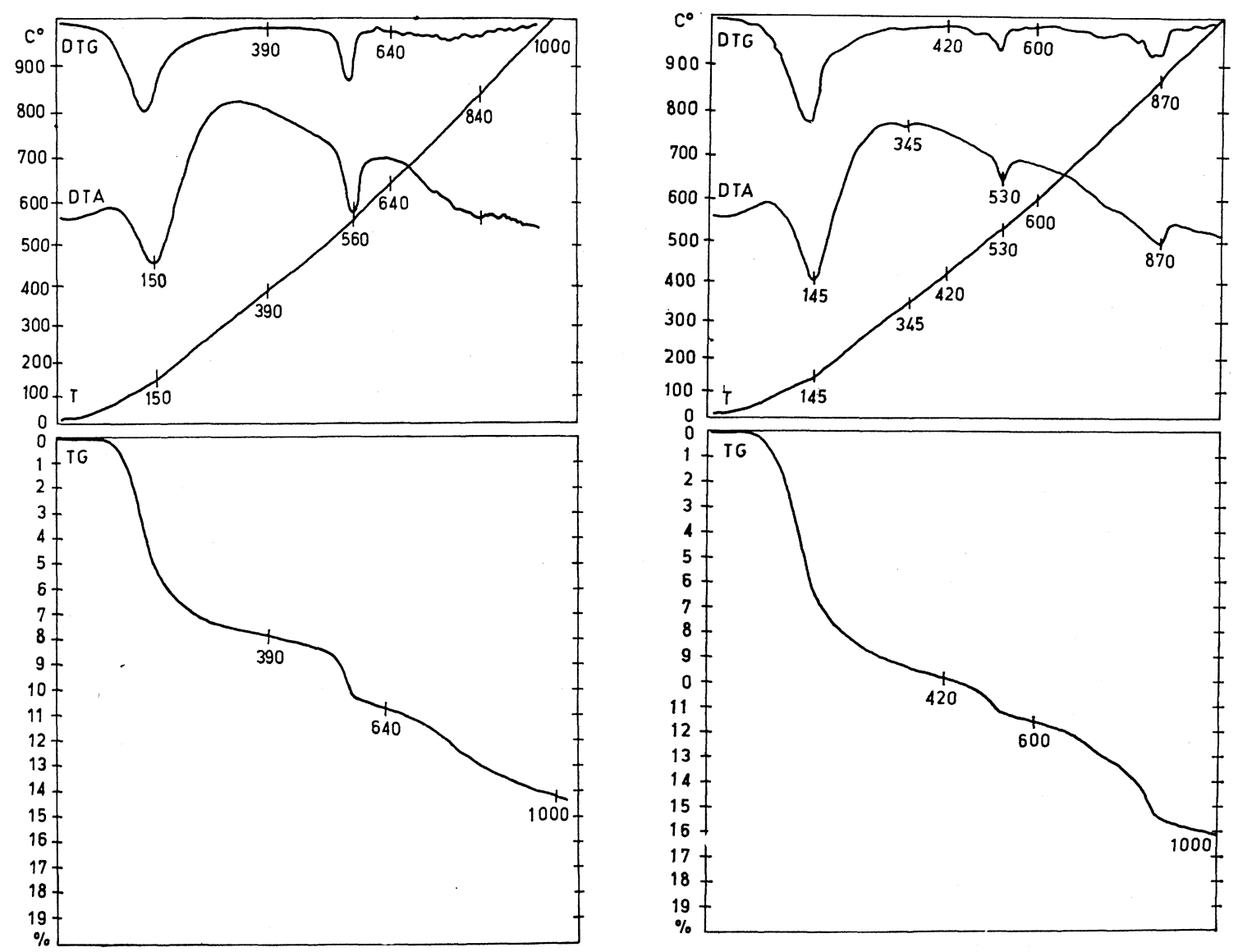

DTA $=$ Termoanálisis diferencial.
DTC $=$ Termogravimetría de derivación.
TC $=$ Termogravimetría.
Fig. 5.-Análisis termográfico del Z II.

Fig. 6.-Análisis termográfico del $\mathrm{Z}$ II con $1,4 \%$ de aditivo.

Todas las mezclas presentan inicialmente efectos endotérmicos perfectamente diferenciados a $145^{\circ}-155^{\circ} \mathrm{C}$ y $530^{\circ}-560^{\circ} \mathrm{C}$. El primero está producido por la ettringita y el segundo por el hidróxido de calcio desintegrado. La influencia de la ettringita es mayor en los cementos con aditivo (figs. 6 y 7), y con el hidróxido cálcico es menor que con el cemento puro (fig. 5). 


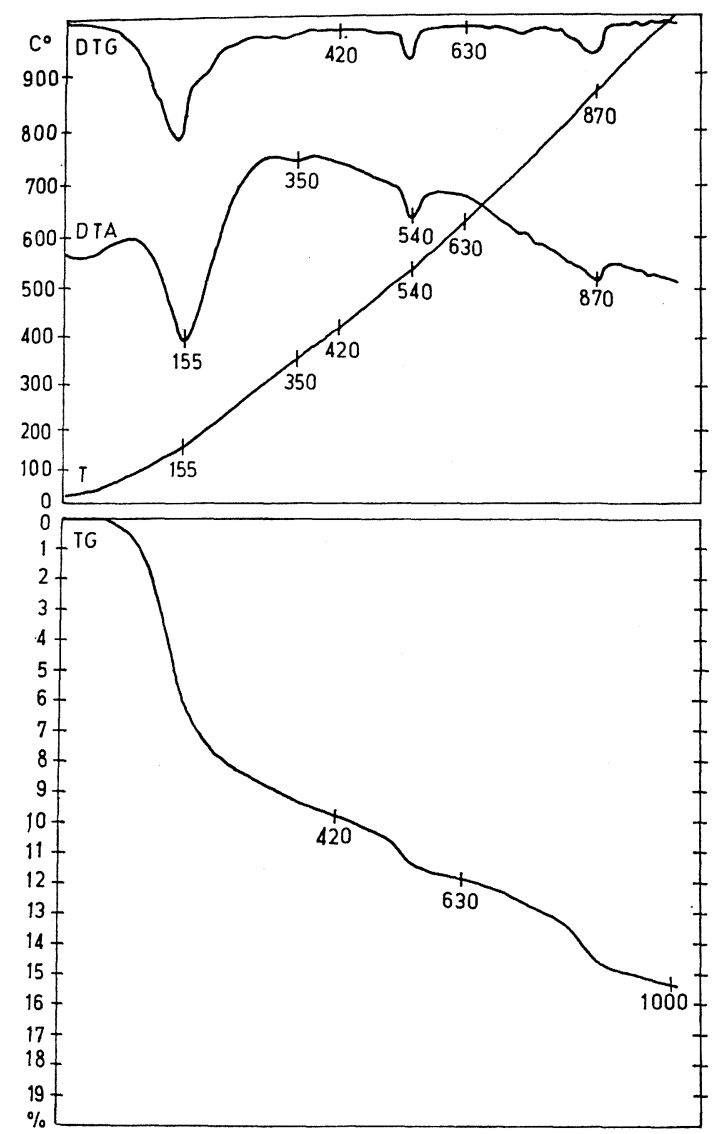

Fig. 7.-Análisis termográfico del $\mathrm{Z}$ III con $2,4 \%$ de aditivo.
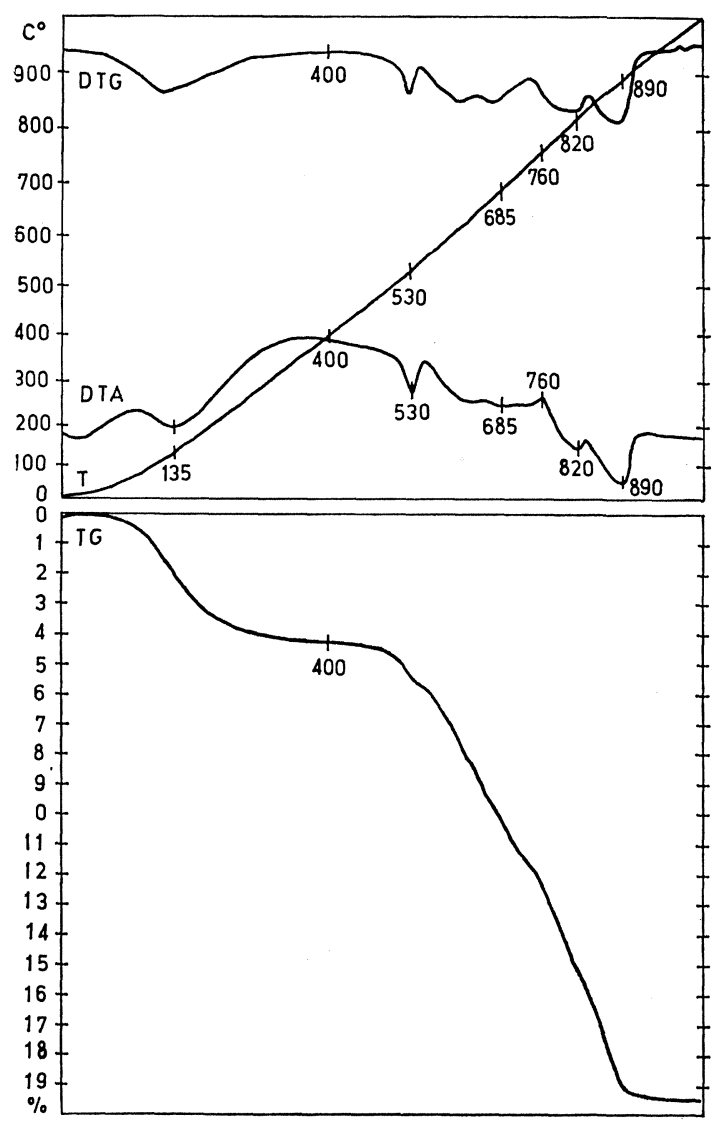

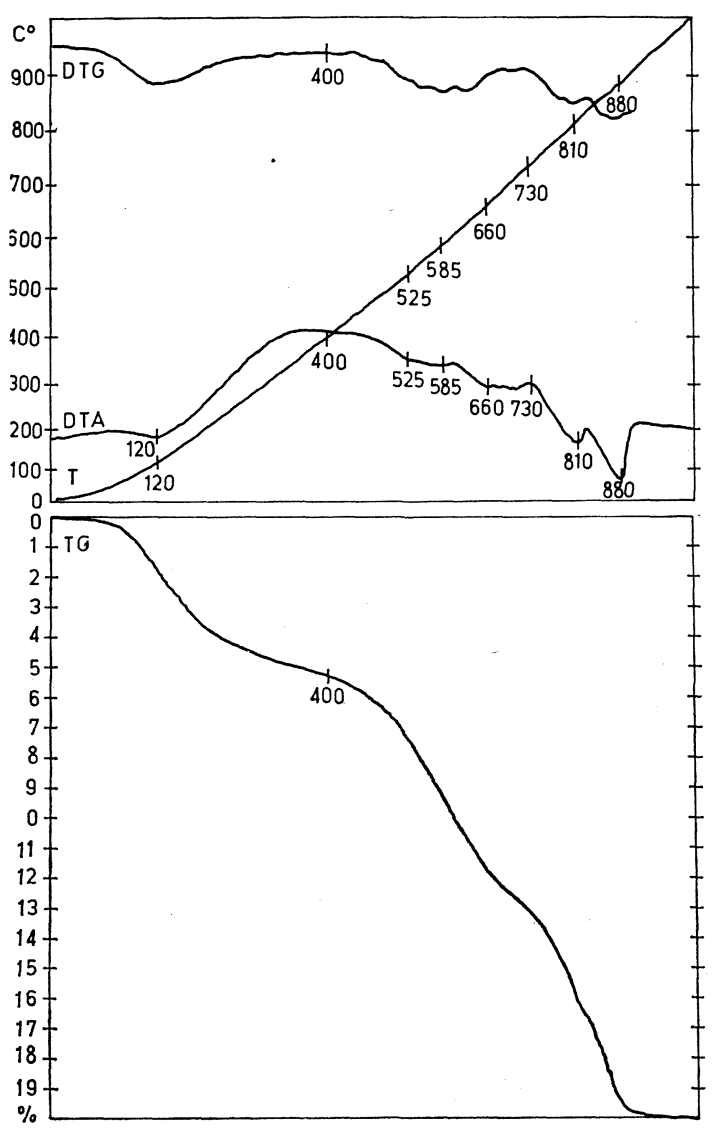

Fig. 8.-Análisis termográfico del $\mathrm{Z}$ III pulverizado al aire, al cabo de un año de curado.

Fig. 9.-Análisis termográfico del $Z$ II con $1,4 \%$ de aditivo pulverizado al aire, al cabo de un año de curado.

Los efectos endotérmicos débiles, de $840^{\circ}$ hasta $870^{\circ} \mathrm{C}$, probablemente deben atribuirse al carbonato cálcico, producido al curar las probetas al aire libre.

Solamente los cementos con aditivo presen$\tan$ un efecto endotérmico débil a $345^{\circ}-350^{\circ} \mathrm{C}$. Puede ser una consecuencia del segundo período de hidratación de la ettringita, aun cuando ésta, según diversos autores, ocurre a una temperatura algo inferior $\left(280^{\circ}\right.$ a $\left.300^{\circ} \mathrm{C}\right)$. Además, los endoefectos a $300^{\circ}-350^{\circ} \mathrm{C}$ los refieren, también, al $\mathrm{C}_{3} \mathrm{AH}_{6}, \mathrm{Al}_{2} \mathrm{O}_{3} \cdot 3 \mathrm{H}_{2} \mathrm{O}$, hidratos de silicato cálcico similares a tobermorita, y según algunas referencias bibliográficas, también al hidrato del monosulfoaluminato. Otros autores afirman que en el caso del hidrato del monosulfoaluminato aparece un efecto endotérmico grande a $200^{\circ}-230^{\circ} \mathrm{C}$. A pesar de la imposibilidad de determinar inequívocamente este efecto, por la diferencia 
con las magnitudes de otros efectos, se ven las grandes variaciones que ocasiona el aditivo en los nuevos compuestos de la hidratación, a pesar de su cantidad reducida. Las diferencias resultan más claras por el examen de los resultados del análisis gravimétrico.

Con ayuda de los campos horizontales de la DTG se determinan los límites de los efectos. Así es posible calcular la reducción de peso de TG de acuerdo con cada efecto aislado. En la tabla 5 figuran los resultados obtenidos.

TAB A A 5

Pérdidas de peso durante el calentamiento

\begin{tabular}{|c|c|c|c|c|c|}
\hline Composición & $\begin{array}{l}\text { Límite superior } \\
\text { del efecto } \\
145^{\circ} \text { a } 155^{\circ} \mathrm{C}\end{array}$ & $\begin{array}{l}\text { Pérdida de peso } \\
\text { del efecto } \\
145^{\circ} \text { a } 155^{\circ} \mathrm{C} \\
(\%)\end{array}$ & $\begin{array}{l}\text { Límite superior } \\
\text { del efecto } \\
530^{\circ} \text { a } 560^{\circ} \mathrm{C}\end{array}$ & $\begin{array}{c}\text { Pérdida de peso } \\
\text { del efecto } \\
530^{\circ} \text { a } 560^{\circ} \mathrm{C} \\
(\%)\end{array}$ & $\begin{array}{l}\text { Pérdida total de } \\
\text { peso hasta } \\
1.000^{\circ} \mathrm{C} \\
(\%)\end{array}$ \\
\hline 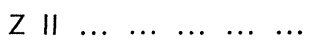 & $390^{\circ}$ & 7,9 & $640^{\circ}$ & 2,9 & 14,2 \\
\hline$z \|+1,4 \%$ adición & $420^{\circ}$ & 9,9 & $600^{\circ}$ & 1,7 & 16,1 \\
\hline Z II $+2,4 \%$ adición & $420^{\circ}$ & 9,8 & $630^{\circ}$ & 2,1 & 15,3 \\
\hline
\end{tabular}

Al comparar las cantidades mayores de agua combinada mediante análisis termogravimétrico, en relación con los compuestos, que se pueden disgregar al calentarse hasta $390^{\circ}$ $420^{\circ} \mathrm{C}$ (ettringita), y las cantidades menores de agua combinada, en relación con los compuestos, que se pueden disgregar al calentarse hasta $600^{\circ}-640^{\circ} \mathrm{C}$ (portlandita), con las cantidades calculadas estoquiométricamente después de la reacción [1], resulta claro que son mayores, especialmente cuando el volumen de aditivo es pequeño. Esto muestra que, además de la formación de ettringita adicional después de la reacción [1], el aditivo cumpuesto, especialmente tratándose de pequeñas cantidades, provoca una hidratación mayor del cemento. Bajo la influencia del aditivo se originan nuevas formaciones de hidratos de los minerales del clínker que pueden disgregarse al calentarlas unos $400^{\circ} \mathrm{C}$ en mayor cantidad que en el cemento puro. Esto se puede comparar en paralelo con los resultados del examen de las resistencias, especialmente en el tiempo inicial.

Las curvas del análisis termográfico, al cabo de un año, aparecen en las figuras 8 y 9 . Como las muestras se conservaron pulverizadas, se pueden diferenciar los resultados bastante bien respecto del tiempo inicial. Al conservarlas al aire, el cemento fraguado molturado se halla completamente sometido a la influencia del secado y del ácido carbónico del aire, que solamente influyen sobre la superficie. Es por lo que las variaciones de las curvas ATD y TG, referidas al tiempo inicial, sólo se aplican a la superficie de hormigón, la cual está sometida a la influencia de la carbonatación y del secado.

En lugar de los grandes efectos endotérmicos de la ettringita a $145^{\circ}-155^{\circ} \mathrm{C}$, a edad inicial, al cabo de un año se presentan efectos considerablemente menores a $120^{\circ}-135^{\circ} \mathrm{C}$, que pueden originarse juntamente con los endotérmicos a $810^{\circ}-820^{\circ} \mathrm{C}$ y los exotérmicos a $730^{\circ}-760^{\circ} \mathrm{C}$ de los hidratos de silicato cálcico. El endoefecto en el caso del cemento con aditivo a $135^{\circ} \mathrm{C}$ es mayor que en el caso del cemento puro. Podría también atribuirse a la ettringita.

Los efectos endotérmicos a $525^{\circ}-585^{\circ} \mathrm{C}$ se pueden atribuir al hidróxido cálcico, y los efectos endotérmicos a $880^{\circ}-890^{\circ} \mathrm{C}$, probablemente al carbonato cálcico. Como era de esperar, los efectos relativos al carbonato cálcico son mayores que en las probetas de edad inicial.

De la curva termogravimétrica derivada se puede determinar solamente un límite a $400^{\circ} \mathrm{C}$, entre los endoefectos a $120^{\circ}-135^{\circ} \mathrm{C}$ y los efectos a temperaturas superiores. 
Al enjuiciar los resultados del análisis termogravimétrico, resulta claro que con el tiempo de un año se han reducido considerablemente las nuevas formaciones de hidratos que se pueden descomponer por debajo de $\operatorname{los} 400^{\circ} \mathrm{C}$. Con el cemento puro la reducción de peso en este campo de temperaturas ha descendido de 7,9 al 5,3\% y en el caso del cemento con aditivo ha retrocedido más, del $9,9 \%$ al 4,3\%. Correlativamente aumenta la pérdida de peso de los compuestos, que se pueden descomponer a temperaturas superiores, y especialmente del carbonato cálcico. Por esta razón también aumentó la pérdida de peso total al calentar hasta $1.000^{\circ} \mathrm{C}$ en relación al cemento puro, de $14,2 \%$ hasta 20,1 $\%$, y en relación al cemento con aditivo, algo menos, de $16,1 \%$ al 19,4 \%.

\section{R E S U MI E N}

Las investigaciones muestran que mediante el empleo combinado de pequeñas cantidades de sulfato de aluminio, junto con cloruro cálcico, se forman cantidades adicionales de ettringita y aumenta el grado de hidratación del cemento. En este caso, la resistencia de un día del cemento aumentó, en comparación con el cemento sin aditivo, del 49 al 125 $\%$, lo que correspondía al 50-67\% de su resistencia, al cabo de 28 días, o del 58 al $70 \%$ de resistencia, al cabo de 28 días, del cemneto sin aditivo. Al pasar el tiempo retrocedió el aumento de resistencia, pero permaneció siempre en un 4-27\% más que en el caso del cemento sin aditivo.

El comienzo del fraguado del cemento se aceleró sensiblemente y asimismo aumentó también sensiblemente el volumen al curar bajo agua y la retracción cuando se cura al aire. El termoanálisis diferencial y las medidas termogravimétricas demuestran la formación de ettringita adicional en función de la cantidad de hidróxido de calcio liberada durante la hidratación del cemento, así como en general una mayor hidratación producida por el aditivo.

Curada al aire la pasta pulverizada de cemento, con y sin aditivo, al cabo de un año acusó variaciones debidas al secado y a la acción del ácido carbónico del aire.

De los resultados de laboratorio se desprende que se puede aplicar el procedimiento para aumentar la resistencia del cemento, sin que para ello sean necesarias especiales inversiones o modificaciones de la tecnología del hormigón.

\section{F I B L I O G R A F I A}

(1) Michaelis, W.: Der Zement-Bazillus. Tonind.-Ztg. 16 (1892), 64.

(2) Berman, H. A. und Newman, E. S.: Chemistry of Cement. Proceeding of the 4th Intern. Sypos., Washington, 1960. Nat. Bur. of Stand., Monograph 43 U.S. Dep. of Commerce (1962), 247.

(3) LaRionowa, S. M.: Die Bildung von Calciumalumint-sulfathydrat...... Wissenchaftlicher Bericht Nr. 5, NIIZB, Gosstroiisdat, Moskau (1959) (russisch).

(4) Turriziani, R., in: H. F. W. TAYlor.: The Chemistry of Ceemnts, Bd. 1, S. 233. Academic Press, London and New York (1964).

(5) Smolczyк, H. G.: Hüttenzemente, Sonderzemente, Chemische Zusätze. Zement-Kalk-Gips 14 (1961), 124. 
(6) Rosemberg, A. M.: Study of the Mechanism through which Calcium Chliride Accelerate the set of Portland Cement. Journ. of the Amer. Concrete Inst. 10 (1964), 1.261.

(7) Tenoutasse, N.: Untersuchungen über die Kinetik der Hydratation des Tricalciumaluminates in Gegenwart von Calciumsulfat und Calciumchlorid. Zement-Kalk-Gips 20 (1967), 459.

(8) Mtchedlov-Petrossian, O. P. und Filatov, L. G.: Quellpusammensetpungen auf Portlandzementbasis. Gosstroiisdat, Moskau (1965) (russisch).

(9) Babuschikin, W. I., Gontovoi, S. W., Dolgowa, O. I., Mokritzkaja, L. P. u. Mtchedlov-Petrossian, O. P.: Komplexzusatz für Quellzementvorbereitung. Patent USSR, Nr. 291895 vom 31.12 .1968 (russisch).

(10) Kutateladse, K. S., Gabadadse, T. G. und Suladse, I. Sch.: Zusätze für schnellerhärtende Zemente. Zement (russisch) 36 (1970), Nr. 8, S. 15.

(11) Gorschkov, W. S.: Thermographie der Baustoffe. Gosstroiisdat, Moskau (1968) (russisch).

(12) Buhlert, R. und Kuzel, H. J.: Über den Einbau von $\mathrm{Cr}^{3+}$ und $\mathrm{Fe}^{3+}$ in Ettringit. Zement-Kalk-Gips 24 (1971), 83.

(13) Englert, G., Wittmann, F. und Nussbaum, M.: Studium der Bindungsverhältnisse des Wassers im Zementstein. Zement-Kalk-Gips 24 (1971), 165.

(14) Boros, M. und BALAzS, Gy.: Verfestigung von $\mathrm{C}_{3} \mathrm{~A}-\mathrm{CaSO}_{4}-\mathrm{H}_{2} \mathrm{O}-$-Systemen bei der Dampfbehandlung. Zement-Kalk-Gips 24 (1971), 473.

(15) Budnikov, P. P., Kusnetzowa, I. P. und Saweliev, W. G.: Chemie und Technologie von Baustoffen und Keramik. Gosstroiisdat, Moskau (1965), 371 (russisch).

(16) TAYlor, H. F. W.: The Chemistry of Cements, IV. Academic Press, London and New York (1964). 